\title{
A Web-Based Cardiovascular Risk Assessment via Pharmacists: A Feasibility and Validation Study
}

\author{
Alexandra de Toledo1,2, Pascal Bonnabry33, Jean-Michel Gaspoz'1, \\ Jean-Philippe de Toldeo' ${ }^{2}$, Idris Guessous ${ }^{1,4,5^{*}}$ \\ ${ }^{1}$ Division of Primary Care Medicine, Department of Community Medicine, Primary Care and Emergency \\ Medicine, University Hospitals of Geneva, Faculty of Medicine, University of Geneva, Geneva, Switzerland \\ ${ }^{2}$ PP Pharmacie Principale SA, Geneva, Switzerland \\ ${ }^{3}$ Pharmacy, Geneva University Hospitals, Geneva, Switzerland; School of Pharmaceutical Sciences, University of \\ Geneva, University of Lausanne, Geneva, Switzerland \\ ${ }^{4}$ Community Prevention Unit, University Institute of Social and Preventive Medicine, Lausanne University \\ Hospital, Lausanne, Switzerland \\ ${ }^{5}$ Department of Epidemiology, Rollins School of Public Health, Emory University, Atlanta, USA \\ Email: ${ }^{*}$ dris.Guessous@hcuge.ch
}

Received 20 July 2015; accepted 18 August 2015; published 21 August 2015

Copyright (C) 2015 by authors and Scientific Research Publishing Inc.

This work is licensed under the Creative Commons Attribution International License (CC BY). http://creativecommons.org/licenses/by/4.0/

(c) (i) 0 pen Access

\section{Abstract}

Background: Involving pharmacists in clinical care could improve the identification of subjects at risk for cardiovascular (CV) disease. Data on web-based approach involving pharmacists for CV disease risk assessment are very limited. Methods: We first developed a web-based CV risk assessment tool to be used by pharmacists that includes demographic, lifestyle, biological and anthropometric information. Biological and anthropometric data were collected in independent laboratories. We then assessed the feasibility and validity of this approach by inviting adults who previously (within 6 months) participated in a Swiss standardized population-based study to fill out the web-based platform. Attrition rates and correlations were used to assess the feasibility and validity, respectively. Proportions were expressed as percentages and continuous variables were expressed as means \pm standard deviations (SD). Main Outcomes Measure: Proportions of participants who 1) agreed to participate; 2) filled out the questionnaire and had their biological and anthropometric measures taken; 3) only filled out the questionnaire; and 4) only had their biological and anthropometric measures taken. Correlations were used to compare continuous variables (body mass index [BMI], waist circumference, systolic blood pressure, fasting blood glucose, total plasma cholesterol, HDL plasma cholesterol, LDL plasma cholesterol, triglycerides) col-

\footnotetext{
${ }^{*}$ Corresponding author.
}

How to cite this paper: de Toledo, A., Bonnabry, P., Gaspoz, J.-M., de Toldeo, J.-P. and Guessous, I. (2015) A Web-Based Cardiovascular Risk Assessment via Pharmacists: A Feasibility and Validation Study. Open Journal of Preventive Medicine, 5, 348-358. http://dx.doi.org/10.4236/ojpm.2015.58039 
lected via both studies. Results: Overall, 218 (53.2\% women) adults of the population-based study were eligible and were contacted to participate, from April to November 2013. Of these, 140 $(64.2 \%)$ agreed to participate. The majority $(67 / 140,47.8 \%)$ both filled out the questionnaire and had their biological/anthropometric measures taken, whereas only $2.8 \%$ and $7.1 \%$ only filled out the questionnaire or only had their biological measures taken, respectively. Except for systolic blood pressure, fasting glucose, and triglycerides, the correlations between the measures obtained in the population-based study and the web-based approach were generally greater than 0.80 , suggesting very good correlations. Conclusions: A web-based CV risk assessment via pharmacists is a feasible and valid approach. This web-based approach should be adapted to lower attrition, and its impact on $\mathrm{CV}$ risk factors should be further tested.

\section{Keywords}

Pharmacists, Web-Based, Cardiovascular Disease, Risk, Feasibility

\section{Introduction}

Cardiovascular (CV) disease is a major cause of mortality and morbidity worldwide [1]. Both behavioral (e.g., unhealthy diet, sedentary lifestyle, psychological stress, smoking, and alcohol consumption) and physiological (e.g., high blood pressure, hypercholesterolemia, and hyperglycemia) factors are associated with CV disease [2]-[4].

Most of these factors are modifiable, and previous studies have highlighted the opportunity to act on these risk factors in order to reduce the likelihood of CV disease. The likelihood of CV disease is generally determined using health risk assessment strategies [5]. So far, the majority of individual CV risk assessment relies on physician involvement. However, this conventional physician-based approach might be suboptimal due, in part, to the shortage of physicians and their lack of time for preventive care.

The management of patients with CV risk factors is currently suboptimal. For example, half of hypertensive subjects remain untreated or uncontrolled [6]. The incidence of CV disease will increase in the next decades, and suboptimal management is likely to increase in parallel [7]. Because the resources providing face-to-face physician-patient interactions are not increasing at the same pace as CV disease and risk factors, innovative methods are required.

Involvement of other healthcare providers such as pharmacists could meaningfully contribute to better identifying subjects at risk for CV disease and to mitigating the CV disease burden by reducing risks through appropriate interventions (e.g., lifestyle changes, suggestion to consult a physician). Pharmacists are freely accessible and knowledgeable healthcare providers [8] with skills and knowledge that can help patients manage important health-related factors and conditions. In addition, evidence from clinical trials supports the use of pharmacist-based interventions to reduce CV risk factors [9]-[17]. Accordingly, in some healthcare systems (e.g., Switzerland), pharmacists are asked by law to influence and motivate their patients to take care of their health [18]. Unfortunately, pharmacists often lack the time required to ask patients all the relevant questions to perform an optimal CV risk assessment. Therefore, new approaches such as web-based questionnaires to complete the assessment generally performed at the counter have been explored.

Accumulating evidence suggests that the use of web-based approaches may also facilitate the promotion of favorable health behavior and the control of CV risk factors [19] [20]. For example, a recent systematic review and meta-analysis found that Internet-based interventions significantly reduced systolic blood pressure in a magnitude comparable to the blood pressure reduction reported in meta-analyses of face-to-face lifestyle counseling [21]. Web-based health risk assessment with tailored feedback for individual health promotion has also emerged [22]. So far, web-based approaches involving pharmacists have generally been limited to controlling drug interactions and/or adherence [23]. Data on the use of web-based approaches specifically involving pharmacists for the prevention, management, or risk assessment of CV disease are limited [24].

Recognizing this, we aimed to determine the feasibility of a web-based CV risk assessment via pharmacists. To do so, we first developed a web-based platform (Health Audit) and then assessed its validity using data from 
the population-based Bus Santé study. We suggest that this web-based CV risk assessment could be used by pharmacists, including at the counter, to contribute-in close collaboration with primary-care physicians-to better identifying subjects at risk for $\mathrm{CV}$ disease and to mitigating their risk by reducing risks through appropriate interventions.

\section{Methods}

\subsection{Health Audit}

We developed a web-based online questionnaire (Health Audit http://www.net-care.ch/) that aims to help individuals manage their own health through a CV risk factor evaluation by pharmacists. The Health Audit was elaborated after a systematic review of the literature on the risk factors and prevention of CV disease [3] [4] (a free demonstration is available at http://www.net-care.ch/). The online electronic questionnaire is based on reliable key indicators that identify and evaluate the risk of developing CV disease [2]-[4]. In addition to demographics, family history, personal medical history, and current treatment information, data pertaining to lifestyle and behavioral and physiological factors was collected to help the patients create a tailored health strategy. Embedded within the Health Audit, a CV risk assessment termed the "Health Score", is calculated according to an algorithm, based on the validated Interheart study [4]. The Health Score combines the following ten key indicators: psychosocial stress, nutrition, physical activity, smoking status, alcohol consumption, fasting glucose, total cholesterol, arterial blood pressure, waist circumference, and oxidation. Health Score points are assigned proportionally to the $\beta$ coefficients of the model, and the score is expressed as a percentage. The score is interpreted as a proxy of the patient's health potential and shows the areas in which the patient could focus on to improve his or her score to approach the ideal score $(100 \%)$. The patient can also request a health counseling session with a pharmacist, who provides tailored advice based on the individual results.

Notably, all information is entered directly into the web-based electronic questionnaire by the participants. If no recent anthropometric and/or biological measures are available, the participants can go to one of the 6 affiliated yet independent laboratories in the State of Geneva (Switzerland), where the necessary biological and anthropometric measures are taken using standardized methods. Affiliations with independent laboratories are aimed to ensure the reproducibility of the measures and the confidentiality of the results.

A description of the methods and protocols used to measure arterial blood pressure, fasting glucose, and lipids (total cholesterol, HDL cholesterol, triglycerides, LDL cholesterol) by these affiliated independent laboratories is presented in supplementary Table 1.

The Health Audit is aimed to be a web-based CV risk assessment that is widely available to public. To ensure confidentiality, two different servers with multiple firewalls are used. The first server is used to send a confirmation email to the participant, who then must fill out the online electronic subscription form. The personal data (surname, first name, address) are saved in the first server. The participants choose a password, allowing them to electronically request their results summary, including the Health Score. The second server is used to anonymously collect the participants' health-related information. The document summarizing the results cannot be associated with the participant, and the information is sent online to the respondent through a secured email. All the collected data are saved for five years and then destroyed. Before this feasibility and validity study conducted from April to November 2013, the web-based approach was pretested in 300 volunteers.

\subsection{Bus Santé Study}

The Bus Santé study has been described elsewhere [6] [25] [26]. Briefly, this cross-sectional population-based study collects information on CV risk factors in the State of Geneva (Switzerland). Subjects are independently selected throughout each year to represent the canton's non-institutionalized adult residents. Eligible subjects are identified using a list of residents established by the local government that includes all potential eligible participants except persons living illegally in the country. Stratified random sampling is used to select participants by gender within each 10-year age stratum, selecting a number of participants that is proportional to the corresponding population distribution. Each participant receives several self-administered, standardized questionnaires. Each participant brings along their filled-in questionnaires, which are checked for correct completion by trained interviewers (a full visit can be watched on http://epidemiologiepopulation.hug-ge.ch/video busSante.html). A 
Table 1. Study-specific protocols and methods for biological and anthropometric measures.

\begin{tabular}{|c|c|c|c|c|}
\hline & \multicolumn{2}{|c|}{ Health Audit study } & \multicolumn{2}{|c|}{ Bus Santé study } \\
\hline & Protocols & Methods & Protocols & Methods \\
\hline BMI $\left[\mathrm{kg} / \mathrm{m}^{2}\right]^{*}$ & Self-reported & $\begin{array}{l}\text { BMI is calculated as } \\
\text { weight }(\text { in } \mathrm{kg} \text { )/height } \\
\left.\text { (in } \mathrm{m}^{2}\right) .\end{array}$ & $\begin{array}{l}\text { Body weight is measured } \\
\text { with the subject lightly } \\
\text { dressed, without shoes } \\
\text { and using a medical } \\
\text { scale (precision } 0.5 \mathrm{~kg} \text { ), } \\
\text { and standing height is } \\
\text { measured using a medical } \\
\text { gauge (precision } 1 \mathrm{~cm} \text { ). }\end{array}$ & $\begin{array}{l}\text { BMI is calculated as } \\
\text { weight (in kg)/height } \\
\text { (in } \mathrm{m}^{2} \text { ). Mean of } \\
3 \text { measures }\end{array}$ \\
\hline Waist circumference [cm] & & $\begin{array}{l}\text { Circumferences to within } \\
5 \mathrm{~mm} \text { with a centimetre } \\
\text { tape, with the subject } \\
\text { standing, } 2 \mathrm{~cm} \text { below } \\
\text { belly button }\end{array}$ & & $\begin{array}{l}\text { Circumferences to within } \\
1 \mathrm{~mm} \text { with a centimetre } \\
\text { tape, with waist mid-way } \\
\text { between the lowest rib } \\
\text { and the iliac crest with } \\
\text { the subject standing at the } \\
\text { end of gentle expiration }\end{array}$ \\
\hline $\begin{array}{c}\text { Systolic blood pressure } \\
\text { [mmHg] }\end{array}$ & $\begin{array}{l}\text { Arterial blood pressure } \\
\text { is measured once in the } \\
\text { sitting position on the } \\
\text { right arm after at least } \\
5 \text { minutes rest using } \\
\text { a standard protocol }\end{array}$ & $\begin{array}{l}\text { Manual measure with } \\
\text { sphygmomanometer } \\
\text { (Lian Classic Spengler) }\end{array}$ & $\begin{array}{l}\text { Arterial blood pressure } \\
\text { is measured thrice in the } \\
\text { sitting position on the } \\
\text { right arm after at least } \\
10 \text { minutes rest using } \\
\text { a standard protocol. }\end{array}$ & $\begin{array}{c}\text { Validated automated } \\
\text { oscillometric } \\
\text { sphygmomanometer } \\
\text { (Omron } \circledR \text { HEM-907, } \\
\text { Matsusaka, Japan). } \\
\text { Mean of } 3 \text { measures }\end{array}$ \\
\hline Fasting glucose $[\mathrm{mmol} / \mathrm{L}]$ & & $\begin{array}{l}\text { Commercially available } \\
\text { enzymatic kits (Roche } \\
\text { Cobas c501, CV 2.5\%) }\end{array}$ & & $\begin{array}{c}\text { Commercially available } \\
\text { enzymatic kits (Bayer } \\
\text { Technicon Diagnostics, } \\
\text { CV } 1.4 \% \text { ) }\end{array}$ \\
\hline Total cholesterol & & $\begin{array}{l}\text { Commercially available } \\
\text { enzymatic kits (Roche } \\
\text { Cobas c501, CV 3.3\%) }\end{array}$ & & $\begin{array}{c}\text { Commercially available } \\
\text { enzymatic kits (Bayer } \\
\text { Technicon Diagnostics, } \\
\text { CV } 1.2 \% \text { ) }\end{array}$ \\
\hline $\begin{array}{l}\text { Lipids } \\
\text { [mmol/L] }\end{array}$ & & $\begin{array}{l}\text { Commercially available } \\
\text { enzymatic kits (Roche } \\
\text { Cobas c501, CV 3.6\%) }\end{array}$ & & $\begin{array}{c}\text { Commercially available } \\
\text { enzymatic kits (Bayer } \\
\text { Technicon Diagnostics, } \\
\text { CV } 1.2 \% \text { ) }\end{array}$ \\
\hline LDL-cholesterol & & $\begin{array}{l}\text { Commercially available } \\
\text { enzymatic kits (Roche } \\
\text { Cobas c501, CV 2.3\%) }\end{array}$ & & $\begin{array}{l}\text { Calculated using the } \\
\text { Friedewald formula }\end{array}$ \\
\hline Triglycerides & & $\begin{array}{l}\text { Commercially available } \\
\text { enzymatic kits (Roche } \\
\text { Cobas c501, CV 2.2\%) }\end{array}$ & & $\begin{array}{c}\text { Commercially available } \\
\text { enzymatic kits (Bayer } \\
\text { Technicon Diagnostics, } \\
\text { CV 1.5\%) }\end{array}$ \\
\hline
\end{tabular}

*only available for 54 participants.

clinical exam is performed, including measuring arterial blood pressure and collecting fasting blood samples to measure serum fasting glucose and lipids. There are no language restrictions; the participants must only be able to understand and answer the questionnaires. The participation rates varied between 58\% and 62\%. The Bus Santé study was approved by the ethical research committee of the Geneva University Hospitals.

Study-specific protocols and methods for biological and anthropometric measures are listed in Supplementary Table 1.

\subsection{Feasibility and Validity of the Health Audit Approach}

To assess the feasibility and validity of the Health Audit approach, we invited subjects who participated in the Bus Santé study within the previous 6 months to use the Health Audit web-based approach. The inclusion crite- 
ria were as follows: Geneva residents, aged 20 to 74 years, and not institutionalized. Internet access and a personal email address were also required to participate. Study participation included the following steps: 1) receiving an invitation by letter and confirmation by phone call; 2) completing the subscription form on the web-based electronic questionnaire; 3) filling out the Health Audit web-based questionnaire (including biological and anthropometric measures taken at specific laboratories); and 4) receiving the results (including the CV risk assessment Health Score) by email. Because we aimed to determine the validity of the biological and anthropometric measures collected via the affiliated independent laboratories proposed in the Health Audit approach, all participants were invited to have fasting blood glucose and lipids measured at these specific laboratories. To be considered complete, each participant had to follow all four steps of the process. The specific independent laboratories were compensated (value of 30 Swiss Francs, 1 Swiss Francs $\approx 1$ US\$) for taking the biological and anthropometric measures, and a gift card (with a value of 25 Swiss Francs) was offered to each participant. All participants gave written informed consent. This feasibility and validation study was approved by the ethical research committee of the Geneva University Hospitals.

\subsection{Statistical Analysis}

To determine the feasibility and validity of the Health Audit web-based approach, the following judgment criteria were set. We first determined the proportions of participants who 1) agreed to participate; 2) filled out the questionnaire and had their biological and anthropometric measures taken; 3) only filled out the questionnaire; and 4) only had their biological and anthropometric measures taken. Based on a previous report [27], we considered a proportion greater than $90 \%$ as reflecting low attrition; otherwise attrition was considered high. Then, we determined correlations between continuous variables (body mass index [BMI], waist circumference, systolic blood pressure, fasting blood glucose, total plasma cholesterol, HDL plasma cholesterol, LDL plasma cholesterol, triglycerides) collected via both studies. Correlations of 0.80 or greater were considered good. Notably, waist circumference but not BMI was measured in the specified independent laboratories. We therefore calculated the BMI using the participants' self-reported weights and heights in the Health Audit questionnaire (BMI = weight/[height $]^{2}$, in $\mathrm{kg} / \mathrm{m}^{2}$ ).

The proportions were expressed as percentages. Continuous variables were expressed as the mean \pm standard deviation (SD). Correlations were based on Pearson's correlation coefficient (r). We determined $a$ priori that a minimum sample size of 60 participants was necessary to have a power $>0.80$ to detect a statistically significant correlation with an alpha error of 0.05 for at least one of the biological or anthropometric measures.

\section{Results}

\subsection{Feasibility of the Health Audit Approach}

The study flowchart is illustrated in Figure 1. Overall, 218 (53.2\% women) Bus Santé study participants were eligible and were contacted to participate in the study. Of these, 140 (64.2\%) agreed to participate. The participation rates were similar between men and women (64.8\% and $63.6 \%$, respectively). Of the 78 subjects not included, 39 (50.0\%) were unreachable, 2 (2.6\%) had no Internet access, and 37 (47.4\%) refused to participate.

Of the 140 participants (mean age $=48.2 \pm 10.9$, 70/140 [50\%] women, median days since Bus Santé study participation = 163.2), $59(42.1 \%)$ did not fill out the questionnaire and did not visit an affiliated independent laboratory to have their biological/anthropometric measures taken. The majority (67/140, 47.8\%) of participants filled out the questionnaire and went to have their biological/anthropometric measures taken, whereas only $2.8 \%$ $(4 / 140)$ or $7.1 \%(10 / 140)$ only filled out the questionnaire or only went to have their biological measures taken, respectively. The Health Score was computed for 56 participants, with a mean of $70.1 \% \pm 8.5$.

\subsection{Validity of the Health Audit Approach}

Information on biological measures (total cholesterol, HDL cholesterol, LDL cholesterol, triglycerides, and fasting glucose), waist circumference, and systolic blood pressure was available for 77 (54.9\%) subjects. BMI data were available for fewer participants $(\mathrm{N}=54)$. With the exception of waist circumference, the means/medians were similar between the Bus Santé study and the Health Audit web-based approach (Supplementary Table 2). The mean waist circumference obtained in the Bus Santé study was lower than that obtained in the Health Audit web-based approach $(87.8 \mathrm{~cm}$ vs $90.3 \mathrm{~cm}$, respectively; p value $=0.03)$. The correlations between 


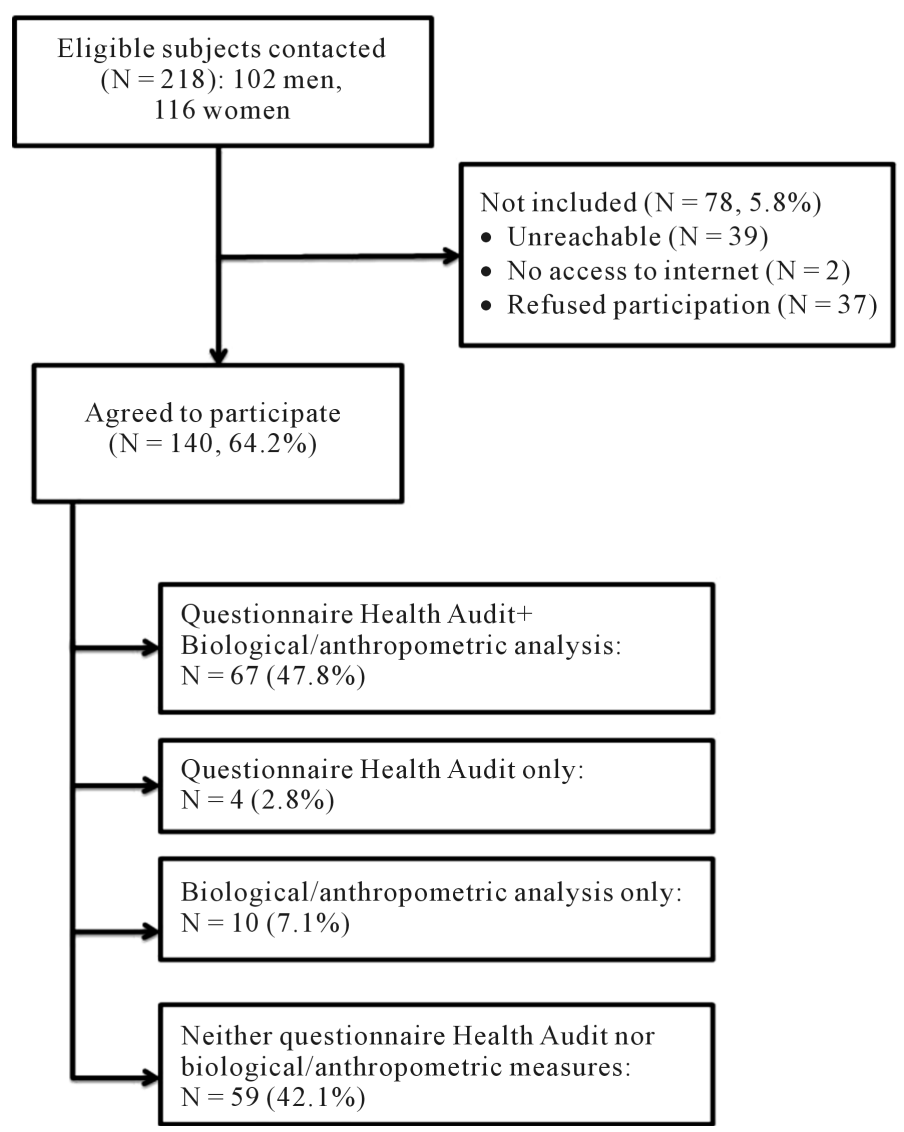

Figure 1. Study flowchart.

Table 2. Means ( \pm standard deviation) of CVD risk factors ( $N=77$ participants), by studies.

\begin{tabular}{cccc}
\hline & Health Audit study & Bus Santé study & P values \\
\hline BMI $\left[\mathbf{k g} / \mathbf{m}^{2}\right]^{*}$ & $24.86 \pm 3.66$ & $24.98 \pm 3.53$ & 0.86 \\
Waist circumference [cm] & $90.35 \pm 10.37$ & $87.84 \pm 9.18$ & 0.12 \\
Systolic blood pressure [mmHg] & $119.81 \pm 13.45$ & $119.66 \pm 16.05$ & 0.95 \\
Fasting glucose [mmol/L] & $5.05 \pm 0.72$ & $5.01 \pm 0.75$ & 0.74 \\
Total cholesterol & $5.30 \pm 1.08$ & $5.58 \pm 1.06$ & 0.11 \\
HDL-cholesterol & $1.51 \pm 0.37$ & $1.41 \pm 0.35$ & 0.09 \\
Lipids [mmol/L] & $3.49 \pm 0.98$ & $3.67 \pm 0.92$ & 0.24 \\
\hline
\end{tabular}

*only available for 54 participants.

the measures obtained in the Bus Santé study and the Health Audit web-based approach were greater than 0.80, suggesting very good correlation between the studies (Figure 2). The correlations for systolic blood pressure, fasting glucose, and triglycerides were below 0.80 .

\section{Discussion}

In this study, we found evidence that a web-based tool evaluated by pharmacists is a feasible approach to generate a CV risk assessment in adults. 


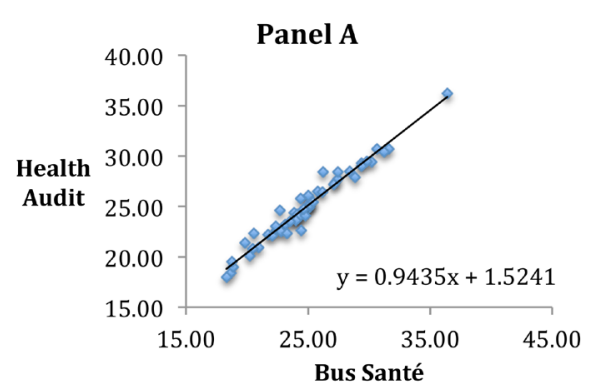

Panel B

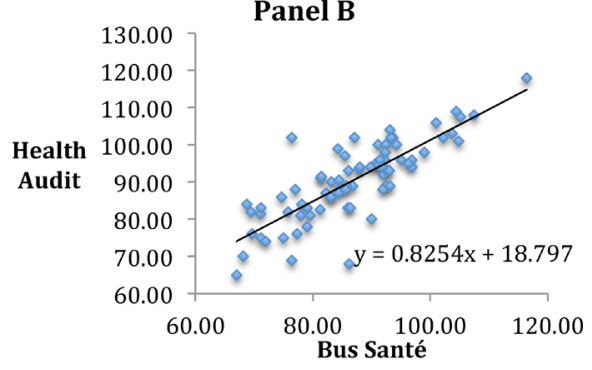

Panel C

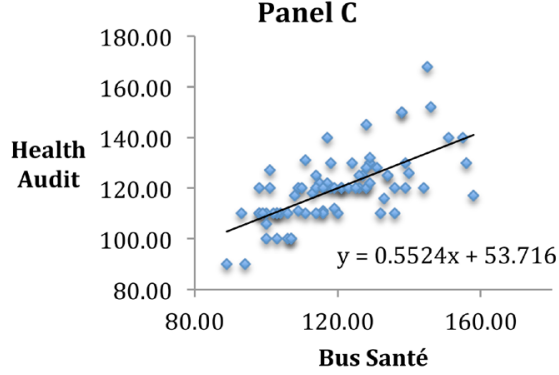

Panel D

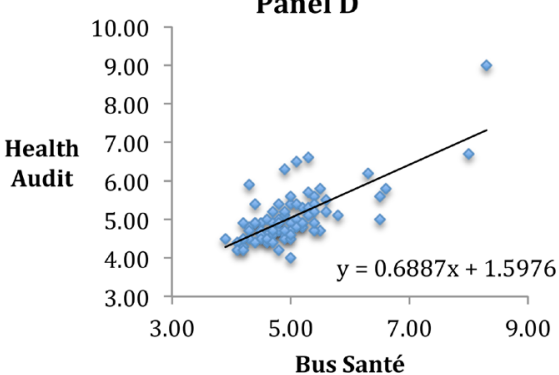

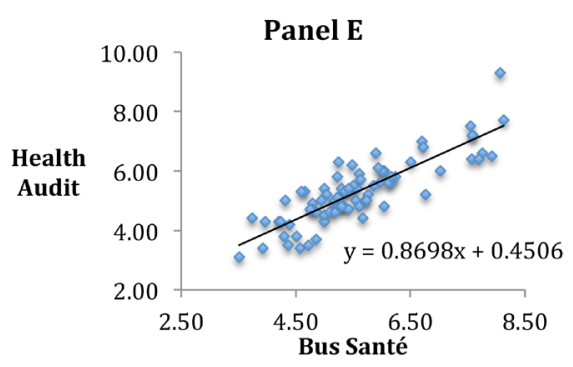
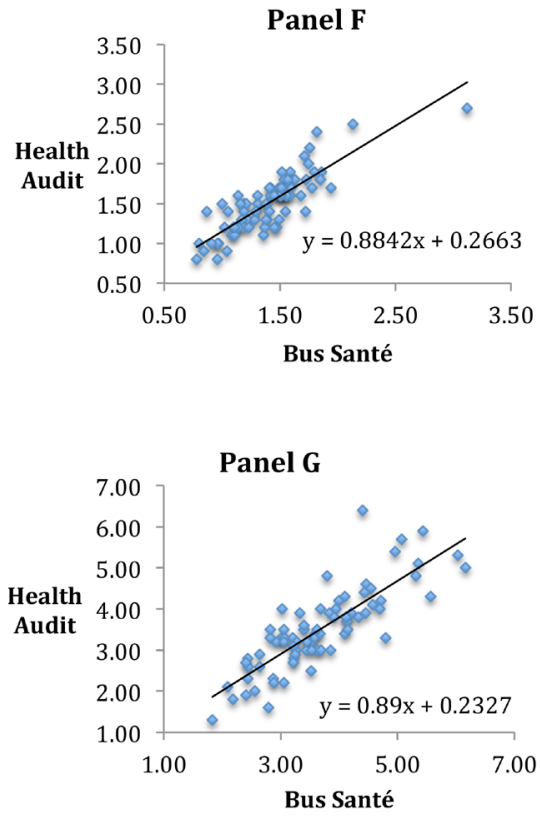

Panel H

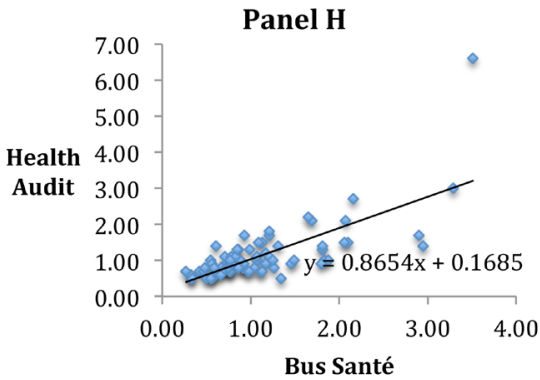

Figure 2. Bus Santé and Health Audit correlation plots, Panel A - H. Footnote: panel A) Body mass index (pearson correlation $r=0.98$, p value $=0.86)$, panel $B$ ) waist circumference $(r=0.77$, p value $=0.12)$, panel C) systolic blood pressure $(r=0.66$, $p$ value $=0.95)$, panel $\mathrm{D})$ glycemia $(r=0.71, \mathrm{p}$ value $=0.74)$, panel E) total cholesterol $(r=0.85$, $p$ value $=0.11)$, panel $F)$ HDL-cholesterol $(r=$ 0.84 , $\mathrm{p}$ value $=0.09)$, panel G) LDL-cholesterol $(\mathrm{r}=0.83$, $\mathrm{p}$ value $=0.24)$, panel $\mathrm{H})$ triglycerides $(\mathrm{r}=$ $0.71, \mathrm{p}$ value $=0.86$ )

Approximately $64 \%$ of subjects who were originally selected at random from the population and contacted for this study agreed to participate. Approximately $40 \%$ of the participants who initially agreed to participate eventually did not fill out the online questionnaire or did not have their biological and anthropometric measures at the specific independent laboratories. Thus, the overall participation rate was only 30.7\% (67/218) for both filling out the online questionnaire and having biological/anthropometric measures taken. High attrition is fre- 
quently reported in studies using web-based approaches; this represents a major limitation of the use of the Internet in chronic health management [27] [28]. Factors that enhance internet participation rates have been reviewed; these factors include pressing health concerns and tailored online communication [27]. It is unlikely that subjects invited to participate in the feasibility and validity study had pressing health concerns. Indeed, these subjects already participated in the Bus Santé study within the previous 6 months, during which they received health status information, including their arterial blood pressure, serum fasting glucose, and lipid profile. This certainly decreased the attractiveness of participating. Although the Health Audit provides an individual CV risk assessment and highlights risk factors that should be considered first, further efforts to better tailor this approach must be made to improve the participation rate. Additionally, to validate the procedure for collecting biological and anthropometric data proposed in the Health Audit in case no recent measures were available, subjects were systematically asked to visit specified affiliated independent laboratories in the State of Geneva. This requirement also further decreased the participation rate.

Only $0.9 \%(2 / 218)$ of the randomly invited participants did not have Internet access. This rate is consistent with data showing that a very high percentage of inhabitants in Switzerland have Internet access. Internet access in Switzerland increased from 61.4\% to 85.2\% between 2002 and 2012 (16\% in 1997) [29]. The same source shows that $60 \%$ of people aged 60 and older have Internet access. Additionally, the effect of socioeconomic disparities on Internet access are decreasing, at least in Western countries [29].

Biological and anthropometric measures obtained using a web-based approach and evaluated by pharmacists correlate well with the measures obtained using standardized procedures from a population-based study. Lower correlations were found for triglycerides, fasting glucose and systolic blood pressure. Several factors can, at least in part, explain these lower correlations. Blood pressure is known to present high intra-individual variability [30], which lowers the correlation. In addition, different protocols were used to measure systolic blood pressure. Although all the biological markers were measured using different methods in the Health Audit and Bus Santé studies, glucose and triglycerides are also highly dependent on diet and fasting. In the Bus Santé study, blood samples were systematically collected between 7.30 am and 10.30 am to favor the fasting state. By contrast, participants in the Health Audit study went to the laboratories for blood sampling at any time throughout the day. It is therefore possible that fasting compliance was lower, as suggested by the slightly elevated glucose and triglyceride values observed in the Health Audit study.

Both opportunities and challenges exist in using the Internet for assessing health risks, promoting healthy lifestyles, and managing health-related conditions. In addition to the possible impact on CV risk factors, an Internetdelivered program has the potential to reduce the number of visits to the doctor and is thus a very cost-effective approach [31].

Still, the impact on CV risk factors of using web-based CV risk assessment involving pharmacists compared to usual care remains to be tested. Although a recent systematic review on the effects on CV risk factors of Internet-delivered multiple modifiable lifestyle interventions complementary to usual care found no evidence of superiority of Internet-based approaches [31], one study (out of 9 identified studies) involving pharmacists stood out with respect to its efficacy. In a 3-group randomized controlled trial in 778 participants with uncontrolled essential hypertension and Internet access in Washington State (USA), Green et al. showed that pharmacist care management delivered through secure Web communications with patients significantly improved blood pressure control in patients with hypertension [24].

Although the integration of pharmacists in the multidisciplinary care of inpatients enhances patient outcomes and is becoming standard practice [32], pharmacists are not systematically involved in the multidisciplinary care of outpatients, notably in terms of CV risk factor prevention and management. The added value of pharmacists to quality-improvement strategies has been reported, notably in the domain of blood pressure management [33] [34]. In Switzerland, authorities predict a shortage of physicians in the 2030s, which will lead to a consultation shortage of 11 million, which is one-third of the total number of medical consultations in 2007 [35]. Similar physician shortages are expected in other countries [36].

Pharmacists and other healthcare providers should be more involved in patient care in the 21st century. By assessing and evaluating risk factors and helping people improve their lifestyle and health behaviors, pharmacists can contribute to improving chronic disease prevention and therefore to reducing healthcare costs [37]-[40]. In addition to being more involved in patient care in the 21st century, pharmacists should also use 21st century tools such as web-based, secured, patient-friendly platforms. Mechanisms by which pharmacists could innovatively provide professional primary care services via a Web-based delivery model have been proposed [41]. 
Web-based pharmacy systems have been shown to prevent serious adverse drug events [23]. However, very few studies have explored the feasibility of using a web-based CV risk assessment performed by pharmacists.

\section{Strengths and Limitations}

This study has several strengths and limitations. Participants were recruited from an ongoing population-based study that randomly selects adult inhabitants of the State of Geneva. Previous studies on web-based health risk assessments have generally included voluntary participants, which may bias the participation rate (because voluntary subjects are more likely to participate). The Health Audit includes a score that can help a patient to focus on a given factor based on his individual priorities. This is in line with evidence suggesting that web-based interventions should be self-guided and tailored to individual priorities [42]. Compared with other web-based approach, the Health Audit combines several interrelated lifestyle risk factors. This is consistent with recent evidence suggesting that multiple lifestyle interventions are more effective than a single intervention [31].

Biological and anthropometric measures were taken throughout the study. Although the cost of the measures is limited (CHF 30.00), participation —at least in the biological and anthropometric measures component of the study —is likely to be lower if these measures are not offered. Additionally, weight and height were measured in the Bus Santé study, whereas they were self-reported in the Health Audit. Comparison (i.e., correlation analysis) of BMI values should therefore be made cautiously.

\section{Conclusion}

Internet and related technologies are innovative tools that can be used for the risk assessment, prevention, and management of CV disease. CV risk assessment by pharmacists seems to be a feasible and valid approach. While this web-based approach should be further adapted to reduce attrition rates, and the impact of this approach on CV risk factors requires further study, we suggest that web-based CV risk assessment could be used by pharmacists, including at the counter, to contribute to better identifying subjects at risk for CV disease and to mitigating their risk by reducing risks through appropriate interventions. Such interventions would include lifestyle changes counseling and primary or secondary CV appropriate prevention. Ideally, this should be made in close collaboration with primary-care physicians.

\section{Acknowledgements}

The Bus Santé study is funded by the Geneva University Hospitals through the General Directorate of Health (Canton of Geneva). The Health Audit is funded by the Pharmacie Principale. The funders had no role in decision to publish. The authors thank the collaborators from the affiliated independent laboratories, Dianalabs/Medisupport, in the State of Geneva, Switzerland.

\section{References}

[1] Fuster, V. and Kelly, B.B. (2010) Promoting Cardiovascular Health in the Developing World: A Critical Challenge to Achieve Global Health. National Academies Press, Washington (DC).

[2] Spruill, T.M. (2010) Chronic Psychosocial Stress and Hypertension. Current Hypertension Reports, 12, 10-16. http://dx.doi.org/10.1007/s11906-009-0084-8

[3] Yusuf, S., Hawken, S., Ounpuu, S., et al. (2004) Effect of Potentially Modifiable Risk Factors Associated with Myocardial Infarction in 52 Countries (the INTERHEART Study): Case-Control Study. Lancet, 364, 937-952. http://dx.doi.org/10.1016/S0140-6736(04)17018-9

[4] Rosengren, A., Hawken, S., Ounpuu, S., et al. (2004) Association of Psychosocial Risk Factors with Risk of Acute Myocardial Infarction in 11,119 Cases and 13,648 Controls from 52 Countries (the Interheart Study): Case-Control Study. Lancet, 364, 953-962. http://dx.doi.org/10.1016/S0140-6736(04)17019-0

[5] Wachira, J.K. and Stys, T.P. (2013) Cardiovascular Disease and Bridging the Diagnostic Gap. South Dakota Medicine. The Journal of the South Dakota State Medical Association, 66, 366-369.

[6] Guessous, I., Bochud, M., Theler, J.M., et al. (2012) 1999-2009 Trends in Prevalence, Unawareness, Treatment and Control of Hypertension in Geneva, Switzerland. PloS One, 7, e39877. http://dx.doi.org/10.1371/journal.pone.0039877

[7] Heidenreich, P.A., Trogdon, J.G., Khavjou, O.A., et al. (2011) Forecasting the Future of Cardiovascular Disease in the United States: A Policy Statement from the American Heart Association. Circulation, 123, 933-944. 
http://dx.doi.org/10.1161/CIR.0b013e31820a55f5

[8] Santschi, V., Chiolero, A., Burnand, B., et al. (2011) Impact of Pharmacist Care in the Management of Cardiovascular Disease Risk Factors: A Systematic Review and Meta-Analysis of Randomized Trials. Archives of Internal Medicine, 171, 1441-1453. http://dx.doi.org/10.1001/archinternmed.2011.399

[9] Garcao, J.A. and Cabrita, J. (2002) Evaluation of a Pharmaceutical Care Program for Hypertensive Patients in Rural Portugal. Journal of the American Pharmaceutical Association, 42, 858-864. http://dx.doi.org/10.1331/108658002762063691

[10] Kraemer, D.F., Kradjan, W.A., Bianco, T.M., et al. (2012) A Randomized Study to Assess the Impact of Pharmacist Counseling of Employer-Based Health Plan Beneficiaries with Diabetes: The EMPOWER Study. Journal of Pharmacy Practice, 25, 169-179. http://dx.doi.org/10.1177/0897190011418513

[11] Planas, L.G., Crosby, K.M., Mitchell, K.D. and Farmer, K.C. (2009) Evaluation of a Hypertension Medication Therapy Management Program in Patients with Diabetes. Journal of the American Pharmacists Association: JAPhA, 49, 164170. http://dx.doi.org/10.1331/JAPhA.2009.08164

[12] Fornos, J.A., Andres, N.F., Andres, J.C., Guerra, M.M. and Egea, B. (2006) A Pharmacotherapy Follow-Up Program in Patients with Type-2 Diabetes in Community Pharmacies in Spain. Pharmacy World \& Science: PWS, 28, 65-72. http://dx.doi.org/10.1007/s11096-006-9003-0

[13] Zillich, A.J., Sutherland, J.M., Kumbera, P.A. and Carter, B.L. (2005) Hypertension Outcomes through Blood Pressure Monitoring and Evaluation by Pharmacists (HOME Study). Journal of General Internal Medicine, 20, 1091-1096. http://dx.doi.org/10.1111/j.1525-1497.2005.0226.x

[14] Paulos, C.P., Nygren, C.E., Celedon, C. and Cárcamo, C.A. (2005) Impact of a Pharmaceutical Care Program in a Community Pharmacy on Patients with Dyslipidemia. The Annals of Pharmacotherapy, 39, 939-943. http://dx.doi.org/10.1345/aph.1E347

[15] Sookaneknun, P., Richards, R.M., Sanguansermsri, J. and Teerasut, C. (2004) Pharmacist Involvement in Primary Care Improves Hypertensive Patient Clinical Outcomes. The Annals of Pharmacotherapy, 38, 2023-2028. http://dx.doi.org/10.1345/aph.1D605

[16] Nola, K.M., Gourley, D.R., Portner, T.S., Gourley, G.K., Solomon, D.K., Elam, M., et al. (2000) Clinical and Humanistic Outcomes of a Lipid Management Program in the Community Pharmacy Setting. Journal of the American Pharmaceutical Association, 40, 166-173.

[17] McKenney, J.M., Slining, J.M., Henderson, H.R., Devins, D. and Barr, M. (1973) The Effect of Clinical Pharmacy Services on Patients with Essential Hypertension. Circulation, 48, 1104-1111. http://dx.doi.org/10.1161/01.CIR.48.5.1104

[18] Swiss Law (2006) Swiss Law 02.01 RK Regulations K 302.01 on the Health Professions of the 22.6.2006—Chaptes III, Article 24. Le pharmacien doit user de son autorité pour inciter le patient à prendre toute mesure propre à la sauvegarde de sa santé [The Pharmacist Should Use Its Authority to Encourage the Patient to Take the Necessary Measures to Stay Healthy].

[19] Vandelanotte, C., Spathonis, K.M., Eakin, E.G. and Owen, N. (2007) Website-Delivered Physical Activity Interventions a Review of the Literature. American Journal of Preventive Medicine, 33, 54-64. http://dx.doi.org/10.1016/j.amepre.2007.02.041

[20] Griffiths, F., Lindenmeyer, A., Powell, J., Lowe, P. and Thorogood, M. (2006) Why Are Health Care Interventions Delivered Over the Internet? A Systematic Review of the Published Literature. Journal of Medical Internet Research, 8, e10. http://dx.doi.org/10.2196/jmir.8.2.e10

[21] Liu, S., Dunford, S.D., Leung, Y.W., Brooks, D., Thomas, S.G., Eysenbach, G., et al. (2013) Reducing Blood Pressure with Internet-Based Interventions: A Meta-Analysis. The Canadian Journal of Cardiology, 29, 613-621. http://dx.doi.org/10.1016/j.cjca.2013.02.007

[22] Colkesen, E.B., Ferket, B.S., Tijssen, J.G., Kraaijenhagen, R.A., van Kalken, C.K. and Peters, R.J.G. (2011) Effects on Cardiovascular Disease Risk of a Web-Based Health Risk Assessment with Tailored Health Advice: A Follow-Up Study. Vascular Health and Risk Management, 7, 67-74. http://dx.doi.org/10.2147/VHRM.S16340

[23] Hamblin, S., Rumbaugh, K. and Miller, R. (2012) Prevention of Adverse Drug Events and Cost Savings Associated with PharmD Interventions in an Academic Level I Trauma Center: An Evidence-Based Approach. The Journal of Trauma and Acute Care Surgery, 73, 1484-1490. http://dx.doi.org/10.1097/TA.0b013e318267cd80

[24] Green, B.B., Cook, A.J., Ralston, J.D., Fishman, P.A., Catz, S.L., Carlson, J., et al. (2008) Effectiveness of Home Blood Pressure Monitoring, Web Communication, and Pharmacist Care on Hypertension Control: A Randomized Controlled Trial. JAMA, 299, 2857-2867. http://dx.doi.org/10.1001/jama.299.24.2857

[25] Guessous, I., Joost, S., Jeannot, E., Theler, J.-M., Mahler, P., Gaspoz, J.-M., et al. (2014) A Comparison of the Spatial Dependence of Body Mass Index among Adults and Children in a Swiss General Population. Nutrition \& Diabetes, 4, 
e111. http://dx.doi.org/10.1038/nutd.2014.8

[26] Guessous, I., Luthi, J.C., Bowling, C.B., Theler, J.-M., Paccaud, F., Gaspoz, J.-M., et al. (2014) Prevalence of Frailty Indicators and Association with Socioeconomic Status in Middle-Aged and Older Adults in a Swiss Region with Universal Health Insurance Coverage: A Population-Based Cross-Sectional Study. Journal of Aging Research, 2014, Article ID: 198603. http://dx.doi.org/10.1155/2014/198603

[27] Schubart, J.R., Stuckey, H.L., Ganeshamoorthy, A. and Sciamanna, C.N. (2011) Chronic Health Conditions and Internet Behavioral Interventions: A Review of Factors to Enhance User Engagement. Computers, Informatics, Nursing: CIN, 29, 81-92. http://dx.doi.org/10.1097/NCN.0b013e3182065eed

[28] McConnon, A., Kirk, S.F., Cockroft, J.E., Harvey, E.L., Greenwood, D.C., Thomas, J.D., et al. (2007) The Internet for Weight Control in an Obese Sample: Results of a Randomised Controlled Trial. BMC Health Services Research, 7, 206. http://dx.doi.org/10.1186/1472-6963-7-206

[29] Union IT. http://www.itu.int/en/ITU-D/Statistics/Pages/stat/default.aspx

[30] Schwartz, G.L., Turner, S.T., Moore, J.H. and Sing, C.F. (2000) Effect of Time of Day on Intraindividual Variability in Ambulatory Blood Pressure. American Journal of Hypertension, 13, 1203-1209. http://dx.doi.org/10.1016/S0895-7061(00)01198-5

[31] Vegting, I.L., Schrijver, E.J., Otten, R.H. and Nanayakkara, P.W.B. (2014) Internet Programs Targeting Multiple Lifestyle Interventions in Primary and Secondary Care Are Not Superior to Usual Care Alone in Improving Cardiovascular Risk Profile: A Systematic Review. European Journal of Internal Medicine, 25, 73-81. http://dx.doi.org/10.1016/j.ejim.2013.08.008

[32] Erstad, B.L., Haas, C.E., O’Keeffe, T., Hokula, C.A., Parrinello, K. and Theodorou, A.A. (2011) Interdisciplinary Patient Care in the Intensive Care Unit: Focus on the Pharmacist. Pharmacotherapy, 31, 128-137. http://dx.doi.org/10.1592/phco.31.2.128

[33] Walsh, J.M., McDonald, K.M., Shojania, K.G., Sundaram, V., Nayak, S., Lewis, R., et al. (2006) Quality Improvement Strategies for Hypertension Management: A Systematic Review. Medical Care, 44, 646-657. http://dx.doi.org/10.1097/01.mlr.0000220260.30768.32

[34] Walsh, J.M., Sundaram, V., McDonald, K., Owens, D.K. and Goldstein, M.K. (2008) Implementing Effective Hypertension Quality Improvement Strategies: Barriers and Potential Solutions. Journal of Clinical Hypertension, 10, 311316. http://dx.doi.org/10.1111/j.1751-7176.2008.07425.x

[35] Swiss State Secretariat for Economics Affairs. http://www.seco.admin.ch/?lang=fr

[36] College AAM. How to Fix the Doctor Shortage. https://www.aamc.org/advocacy/campaigns and coalitions/fixdocshortage/

[37] Houle, S.K., Chuck, A.W., McAlister, F.A. and Tsuyuki, R.T. (2012) Effect of a Pharmacist-Managed Hypertension Program on Health System Costs: An Evaluation of the Study of Cardiovascular Risk Intervention by PharmacistsHypertension (SCRIP-HTN). Pharmacotherapy, 32, 527-537. http://dx.doi.org/10.1002/j.1875-9114.2012.01097.x

[38] Cote, I., Gregoire, J.P., Moisan, J., Chabot, I. and Lacroix, G. (2003) A Pharmacy-Based Health Promotion Programme in Hypertension: Cost-Benefit Analysis. PharmacoEconomics, 21, 415-428. http://dx.doi.org/10.2165/00019053-200321060-00005

[39] Munroe, W.P., Kunz, K., Dalmady-Israel, C., Potter, L. and Schonfeld, W.H. (1997) Economic Evaluation of Pharmacist Involvement in Disease Management in a Community Pharmacy Setting. Clinical Therapeutics, 19, 113-123. http://dx.doi.org/10.1016/S0149-2918(97)80078-1

[40] Strong, D.K. and Tsang, G.W. (1993) Focus and Impact of Pharmacists’ Interventions. The Canadian Journal of Hospital Pharmacy, 46, 101-108.

[41] Alston, G.L. and Waitzman, J.A. (2013) The I-Tribe Community Pharmacy Practice Model: Professional Pharmacy Unshackled. Journal of the American Pharmacists Association: JAPhA, 53, 163-171. http://dx.doi.org/10.1331/JAPhA.2013.12199

[42] Noar, S.M. (2012) eHealth Applications: Promising Strategies for Behavior Change. Routledge, New York. 